
\title{
3 Research Square \\ High Expression of RIPK2 is Associated with Taxol Resistance in Serous Ovarian Cancer
}

\section{Yuqing Shen}

Zhejiang University School of Medicine Women's Hospital

\section{Hui Lin}

Zhejiang University School of Medicine Women's Hospital

\section{Kelie Chen}

Zhejiang University School of Medicine

\section{Wanzhong Ge}

Zhejiang University School of Medicine Women's Hospital

\section{Dajing Xia}

Zhejiang University School of Medicine

\section{Yihua Wu}

Zhejiang University School of Medicine

Weiguo Lv ( $\sim$ lbwg@zju.edu.cn )

Zhejiang University School of Medicine Women's Hospital https://orcid.org/0000-0003-2062-7145

\section{Research}

Keywords: serous ovarian cancer, taxol resistance, RIPK2, bioinformatics, immune infiltration

Posted Date: February 26th, 2021

DOI: https://doi.org/10.21203/rs.3.rs-247016/v1

License: (c) (i) This work is licensed under a Creative Commons Attribution 4.0 International License.

Read Full License 


\section{Abstract}

\section{Background}

Taxol resistance of serous ovarian cancer is responsible for its poor prognosis, yet the underlying mechanism was still poorly understood. Thus, we probed the mechanism of taxol resistance in serous ovarian cancer with multiple bioinformatic methods to provide novel insights for potential therapy.

\section{Methods}

The differentially expressed genes (DEGs) and their relationship with overall survival (OS) and progressfree interval (PFI) of ovarian cancer patients were analyzed using gene expression datasets from the Cancer Genome Atlas (TCGA) and Gene Expression Omnibus (GEO). The role of receptor interacting serine/threonine kinase 2 (RIPK2) was validated by identifying its co-expressed genes, making function analysis and generating protein-protein network (PPI). Single-sample GSEA (ssGSEA) method was used to explore the immune infiltration and genomic alterations of RIPK2 was also analyzed via cBio Cancer Genomics Portal (cBioProtal).

Results

RIPK2 was highly expressed in taxol resistant ovarian cancer cell lines, while its high expression was also linked with OS and PFI in serous ovarian cancer patients. The PPI network analysis and pathway analysis demonstrated that RIPK2 might take part in positive regulation of NF-KB transcription factor activity. Different expression level of RIPK2 was related to tumor microenvironment alteration, which might participate in formation of taxol resistance.

\section{Conclusions}

Our studies suggested that high expression of RIPK2 was related to taxol resistance in serous ovarian cancer, while RIPK2 induced taxol resistance through NOD1/RIPK2/NF-KB inflammatory pathway activation and tumor microenvironment changes.

\section{Background}

Serous ovarian cancer (SOC) accounts for $70 \%$ of all ovarian cancers and is known as the most common subtype of ovarian cancer(1). It is composed of high-grade serous ovarian cancer (HGSOC) and lowgrade serous ovarian cancer (LGSOC), while HGSOC(2) has the highest mortality. Ovarian cancer is conventionally treated with surgery and paclitaxel/carboplatin combination chemotherapy(3).Although patients may response to chemotherapy well initially, the 5-year survival rate is still low because of the combination of late stage diagnosis, disease heterogeneity and drug resistance(4).

Taxol is recommended along with platinum as the first-line chemotherapeutic agent against ovarian cancer(5). However, the majority of patients may suffer from disease recurrence and chemoresistance 
during the treatment. Recent studies revealed that taxol resistance may be caused by a series of modifications, including tumor microenvironment changes, pharmacokinetics alterations, changes in signaling pathways, P-glycoprotein (P-gp)upregulation, tubulin dynamic alterations, $\beta$-tubulin gene or expression of $\beta$-tubulin isotype mutations and apoptotic mechanism change(6). Alteration of gene expression levels also plays a significant role in the development of taxol resistance. For example, high expression of tubulin beta 3 class III (TUBB3) and low expression of salt inducible kinase(SIK2), polo-like kinase 2(PLK2) or spleen tyrosine kinase(SYK) restore paclitaxel sensitivity of ovarian cancer cells $(7,8$, 9). Still, taxol resistance in ovarian cancer is much less known and more attention should be paid.

Bioinformatics is a fast-developing method being used widely in cancer related studies, whose application causing the emergence of a great number of literatures focusing on the cancer chemoresistance and recurrence-related genes. Radosław Januchowski et al. (10)used microarray analysis and observed upregulation of ATP binding cassette subfamily $B$ member 1 ( $A B C B 1), E P H$ receptor $\mathrm{A} 7$ ( $E P H A 7)$ and RUN domain containing 3B (RUNDC3B) and downregulation of Endothelial lipase (LIPG), multiple $C 2$ and transmembrane domain containing 1 (MCTP1), high mobility group nucleosome binding domain 5 (HMGN5), protocadherin 9 ( $P C D H 9)$, protein tyrosine phosphatase receptor type $\mathrm{K}$ (PTPRK) and semaphorin 3A (SEMA3A) in paclitaxel-resistant cell lines. Yi Hu et al.(11) found that high Stratifin (SFM) expression is associated with significantly worse overall survival in patients receiving gemcitabine, taxol, taxol+platin, paclitaxel or avastin chemotherapy. Besides, Reto S Kohler et al.(12) reported that elevated maternal embryonic leucine zipper kinase (MELK) expression was correlated to poor survival and taxol resistance in ovarian cancer. However, there is still a lack of research on taxol resistance in ovarian cancer using bioinformatics methods as tools.

In this study, we used Gene Expression Omnibus database to define differentially expressed genes in taxol-sensitive and -resistant ovarian cancer cell lines. The Cancer Genome Atlas (TCGA) and Gene Expression Omnibus (GEO) database were used to determine the influence of selected genes on patients' progression free interval (PFI) and overall survival (OS). Our analysis revealed that high expression of RIPK2 indicated poor PFI and OS. Further research on mechanisms of the relationship between taxol resistance and high expression of RIPK2 was made using function analysis, pathway analysis, Proteinprotein network and cBio Cancer Genomics Portal (cBioPortal) online tools. Together, our study suggested that RIPK2 could act as a biomarker for taxol treatment sensitivity in serous ovarian cancer and might provide new insights into the mechanisms underlying taxol resistance in serous ovarian cancer.

\section{Materials And Methods}

\subsection{Identification of DEGs.}

GEO datasets GSE58840, GSE60335, GSE58878, GSE26465, GSE73935 and GSE54772 were downloaded using R package "GEOquery"(13). R package "limma" was used to identify DEGs in each dataset, while heatmap was drawn using "heatmap" package(14). Difference with $p<0.05$ and $\left|\log _{2} \mathrm{FC}\right|>1$ were 
considered statistically significant. The intersections of DEGs from different datasets were shown using a Venn diagram by R package “VennDiagram”(15).

\subsection{Survival analysis.}

The ovarian cancer gene expression profiles of frozen ovarian cancer tissue samples from 3 cohorts in GEO and 1 cohort from TCGA-OV were selected for survival analysis. Patients selected for our analysis were diagnosed with serous ovarian cancer and received taxol chemotherapy, while their clinical features including overall survival and progression free interval should be available. Survival analysis and twotalied log-rank test (OS and $\mathrm{PFI}$ ) were carried out to compare the certain gene outcome in high and low expression groups using R package "survival"(16). Survival curves were generated by package "survminer"(17). The cutoff values between high and low expression groups were calculated with maximally selected rank and statistics method by R package "survminer". The study characteristics of selected cohorts were described in Table 2.

\subsection{Correlation Analysis}

Correlation of gene expression and taxol resistance was analyzed using Cancer Cell Line Encyclopedia (CCLE) database which contains ovarian cancer cell line gene expression as well as IC50 of taxol(18). Correlation of gene expression was analysed in each dataset, including TCGA-OV, GSE30161, GSE32063 and GSE63885. The analysis was applied using Spearman correlation analysis by R package "corrplot"(19). Correlation coefficients with $p<0.05$ were considered to be statistically significant.

\subsection{Functional pathway enrichment analysis.}

Gene Ontology (GO) consortium provides descriptions of the biological process (BP), cellular component (CC) and molecular function (MF) of a gene list and KEGG (Kyoto Encyclopedia of Genes and Genomes) is a database resource that integrates genomic, chemical and systemic functional information. To understand the function of RIPK2 co-expressed genes, we applied GO and KEGG analysis with R package "clusterProfiler"(20). The bubble map of top significant pathways was drawn using R package "ggplot2" based on $p$ value. $p<0.05$ was set as the cut-off criteria(21).

\subsection{PPI network.}

Protein-protein interaction network (PPI) was used to describe interactions between proteins, providing a deep understanding of cell physiology. We generated PPI network using online tool STRING(22). The obtained PPI interactions were visualized by Cytoscape (version 3.4.0, http://www.cytoscape.org/) (23). 


\subsection{Evaluation of immune infiltration.}

The infiltration of 67 types of immune cells in ovarian cancer samples was evaluated with $\mathrm{R}$ package "xCell" using ssGSEA (single-sample GSEA) method(24). The correlation between immune infiltration and RIPK2 expression was assessed by Spearman analysis and difference with $p<0.05$ was considered as significant. Barplot was generated by "ggplot2" to visualize the correlation coefficients and $P$ value.

\subsection{Genetic alterations analysis.}

The cBioPortal (http://cbioportal.org) contains multiple cancer genomics datasets, including mutation, copy number variation (CNV), and gene co-occurrence information(25). IDs of Patients who were treated with taxol in TCGA-OV dataset were imported to the online cBioPortal tool and RIPK2 alteration was analyzed and visualized. The tab OncoPrint displays an overview of genetic alterations per sample in PIPK2. The alterations and mutations of genes co-expressed with RIPK2 was presented with boxplots generated by cBioportal. Difference with $p<0.05$ was considered to be statistically significant.

\section{Results}

\subsection{Identification of DEGs using GEO datasets}

GEO datasets GSE58840, GSE60335, GSE58878, GSE26465, GSE73935 and GSE54772, containing expression profiles of taxol-sensitive and resistant cell lines, were downloaded using $\mathrm{R}$ package "GEOquery". The study characteristics and sizes of selected datasets were described in Table 1. 226 upregulated genes and 214 down-regulated genes were found in taxol-resistant SKOV3 cells in microarray GSE58840, GSE60335 and GSE58878 (Figure 1A and Supplementary Table 1), while 494 up-regulated genes and 451 down-regulated genes were identified in taxol-resistant A2780 cell line in microarray GSE26465 (Figure 1B and Supplementary Table 1). 150 DEGs were screened from the GSE73935 dataset, including 71 up-regulated genes and 79 down-regulated genes in taxol-resistant OV90 cell line (Figure 1C and Supplementary Table 1). Additionally, 74 and 48 genes were up-regulated and down-regulated respectively in taxol-resistant SKOV3 cell line in dataset GSE54772 (Figure 1D and Supplementary Table 1). The overlapping up-regulated and down-regulated genes were obtained from the intersection of DEG datasets identified above (Figure 1E-F).

Table 1. mRNA sequencing datasets containing taxol-sensitive/resistant cell lines 


\begin{tabular}{|c|c|c|c|c|c|c|}
\hline \multirow{2}{*}{\multicolumn{2}{|c|}{$\begin{array}{l}\text { Accession number of } \\
\text { the dataset }\end{array}$}} & \multirow{2}{*}{ Platforms } & \multirow{2}{*}{ Reference } & \multirow{2}{*}{ Cell lines } & \multicolumn{2}{|c|}{ Response to chemotherapy } \\
\hline & & & & & Sensitive & Resistant \\
\hline Bind & $\begin{array}{l}\text { GSE58840 } \\
\text { GSE60335 } \\
\text { GSE58878 }\end{array}$ & GPL16951 & $\begin{array}{c}\text { Oncotarget } \\
2014 \text { Dec 15;5(23):11939-56. PMID: } 25460502\end{array}$ & SKOV3 & 2 & 4 \\
\hline \multicolumn{2}{|c|}{ GSE26465 } & GPL6140 & $\begin{array}{c}\text { J Ovarian Res } \\
\text { 2011 Dec 5;4(1):21. PMID: } 22141344\end{array}$ & A2780 & 2 & 4 \\
\hline \multicolumn{2}{|c|}{ GSE73935 } & GPL13667 & Oncol Rep 2014 Nov;32(5):1981-90. PMID: 25199881 & ov & 2 & 2 \\
\hline \multirow{2}{*}{\multicolumn{2}{|c|}{ GSE54772 }} & \multirow{2}{*}{ GPL570 } & Mol Oncol & \multirow[t]{2}{*}{ SKOV3 } & 3 & 6 \\
\hline & & & 2015 Apr;9(4):791-805. PMID: 25560085 & & 3 & 3 \\
\hline
\end{tabular}

\subsection{Survival analysis}

To explore if DEGs identified in taxol-sensitive and -resistant ovarian cancer cell lines are related to PFI and OS of ovarian cancer patients, samples from TCGA-OV with recurrence and therapy information are selected and analyzed (Supplementary Table 2). To each DEG identified, correlation of the expression of this gene with PFI and OS was evaluated with Kaplan-Meier method. Samples were divided into a highexpression group and a low-expression group according to the cutoff value of the certain gene, which was calculated by maximally selected rank and statistics method using R package "survminer". For genes that show statistically significant differences in OS and PFI survival analysis, we determined whether the difference of their expression in sensitive and drug-resistant cell lines were consistent with the differences shown in survival analysis. If a specific gene had a higher expression level in taxol-resistant cell line, the survival of patients with this highly expressed gene should be poorer. Interferon Stimulated Gene 15 (ISG15), synuclein alpha (SNCA) and RIPK2 were up-regulated in taxol-resistant cell lines, while their high expression were also correlated with shorter OS and PFI in TCGA-OV dataset. Phospholipase $\mathrm{C}$ gamma 2 (PLCG2), ras homolog family member $\mathrm{U}(R H O U)$, tribbles pseudokinase 2 (TRIB2) and elongator acetyltransferase complex subunit 3 (ELP3) had low expression in taxol-resistant cell lines and their high expression was related to better survival in TCGA-OV dataset (Figure S1-6).

Dataset GSE30161, GSE32062 and GSE63885 which contained clinical information of patients with serous ovarian cancer including OS and PFI were selected to further validate effects of the expression of ISG15, SNCA, RIPK2, PLCG2, RHOU, TRIB2 and ELP2 on patients' chemosensitivity to taxol treatment (Table 2 and Supplementary Figure 1-6). In all three datasets, high expression group and low expression group of RIPK2 showed significant difference in survival analysis with both OS and PFI, suggesting that high expression of RIPK2 was a risk factor of survival in patients with serous ovarian cancer (Figure 2).

We further validated the prediction value of RIPK2 expression by dividing TCGA-OV cohort into four groups based on patients' RIPK2 expression level and if taxol was used during treatment. Survival analysis were carried out and we found that patients with low RIPK2 expression and taxol treatment showed the longest OS, while those with low RIPK2 but were not treated by taxol showed the shortest OS. Meanwhile, there were no significant difference of OS in patients with high expression of RIPK2, no matter they were treated by taxol or not. 
Table 2. mRNA sequencing datasets containing overall survival and progress free interval of serous ovarian patients treated with taxol

\begin{tabular}{|c|c|c|c|}
\hline $\begin{array}{l}\text { Accession number of } \\
\text { the dataset }\end{array}$ & Platforms & Reference & Sample size \\
\hline TCGA-OV & / & / & 254 for OS; 145 for PFI \\
\hline GSE30161 & GPL570 & Plos One 2012;7(2):e30550. PMID: 22348014 & 47 \\
\hline GSE32063 & GPL6480 & $\begin{array}{c}\text { Clin Cancer Res } 2012 \text { Mar 1;18(5):1374-85. } \\
\text { PMID: } 22241791\end{array}$ & 40 \\
\hline GSE63885 & GPL570 & $\begin{array}{l}\text { Front Oncol 2014;4:6. PMID: 24478986; } \\
\text { J Cancer Res Clin Oncol } 2016 \text { Jun;142(6):1239- } \\
\text { 52. PMID: } 27028324\end{array}$ & 36 \\
\hline
\end{tabular}

\subsection{Correlations of RIPK2 gene expression with taxol resistance in CCLE}

CCLE contains a large panel of human cancer cell lines and their pharmacological profiles, in which the gene expression files and IC50 values to taxol of 21 ovarian cancer cell lines were included (Supplementary table 3). By dividing the expression level of RIPK2 in each cell line by the expression level of GAPDH in the same cell line, the expression of RIPK2 was normalized. Correlation of RIPK2 expression and IC50 of taxol was analyzed by R package "corrplot" with Spearman method. The correlation coefficient was $0.46(p<0.05)$, indicating that higher expression of RIPK2 elevated taxol resistance of multiple ovarian cancer cell lines.

\subsection{RIPK2 co-expression network in ovarian cancer}

In order to gain further insight of the biological function of RIPK2 in development of taxol resistance in ovarian cancer, the co-expression mode of RIPK2 in serous ovarian cancer patients treated with taxol was analyzed. In dataset TCGA-OV, 341 genes were found to show a significant positive co-expression pattern with RIPK2, while no gene showed a negative co-expression. The expression of 704 genes had a positive correlation with RIPK2 expression in GSE30161 and 1706 had a negative correlation. 45 genes positively co-expressed while 37 negatively co-expressed with RIPK2 in GSE32063. 17 genes were found in dataset GSE63885 to have a positive co-expression pattern with RIPK2 and 4 genes had a negative co-expression pattern. A total description of the co-expressed genes was detailed in Supplementary Table 4.

Function analysis were made using the intersections of every two datasets as input. Significant GO term showed that RIPK2 co-expressed genes from multiple datasets participate mainly in cell adhesion molecule binding, positive regulation of cytokine production and focal adhesion(Figure 3A-C). KEGG pathway analysis showed an enrichment in NF-kappa B signaling pathway, NOD-like receptor signaling pathway and Ubiquitin mediated proteolysis pathway. (Figure 3D and Supplementary Table 5). 
A PPI network of RIPK2 related genes were created on the basis of information from the STRING database, which further illustrates the connection of these co-expressed genes from the protein level. The average aggregation coefficient was 0.508 , and the enrichment $p<1.0 \mathrm{e}-3$ (Figure $3 \mathrm{E}$ ).

\subsection{Genomic alterations of RIPK2 in ovarian cancer}

cBioPortal tool was used to determine the alterations of RIPK2 in ovarian cancer patients who were treated with taxol in TCGA-OV database. Alterations occurred in 26 of 252 samples (10\%), including 1 missense mutation (0.4\%), 7 amplification (3\%), 21 mRNA upregulation (8\%) and 4 mRNA downregulation (2\%) (Figure 4A). RIPK2 amplification results of high expression of RIPK2, which may relate to taxol resistance. Thus, AMP is the most common type of RIPK2 copy number alteration (CNA) in ovarian cancer (Figure 4B). Meanwhile there was significant difference of amplification of oxidative stress induced growth inhibitor family member 2 (OSGIN2), nibrin (NBN), Ras-Related Protein Rab-2A (RAB2A) and calbindin 1 (CALB1), etc in RIPK2-altered and -unaltered group (Figure 4C and Supplementary Table 6). Moreover, the mutation frequency of ArfGAP with SH3 domain, ankyrin repeat and PH domain 1 (ASAP1), ATP/GTP binding protein 1 (AGTPBP1), frizzled class receptor 7 (FZD7), HECT and RLD domain containing E3 ubiquitin protein ligase 5 (HERC5), KIAA0232, Mitogen-Activated Protein Kinase Kinase Kinase 10 (MAP3K10), PATJ crumbs cell polarity complex component (PATS), PDGFA associated protein 1 (PDAP1), xin actin binding repeat containing 1 (XIRP1) was significantly associated with the alteration of RIPK2 (Figure 4D and Supplementary Table 7).

\subsection{Evaluation the difference of immune cell infiltration}

The immune infiltration of 64 types of immune cells, including adaptive and innate immune cells, hematopoietic progenitors, epithelial cells, and extracellular matrix cells, were evaluated by R package "xCell", using the method of ssGSEA in ovarian cancer tissues. In dataset TCGA-OV, the infiltration of Macrophages M1, Melanocytes and plasmacytoid dendritic cells (pDC) was positively related to the expression of RIPK2, while the infiltration of Neurons was negatively related to the expression of RIPK2 (Figure 5A and Supplementary Table 8-9). Meanwhile, CD8+ naive T-cells, common lymphoid progenitors (CLP), CD4+ memory T-cells, smooth muscle and Hematopoietic stem cells (HSC) infiltrated more when RIPK2 expression levels were higher, but immature dendritic cells (iDC), Neurons, Basophils, Classswitched memory B-cells, mensenchymal stem cells(MSC), microvascular endothelial cells, natural killer T-cells(NKT), pro B-cells, Pericytes, Melanocytes, Mast cells, CD4+ T-cells, Plasma cells, MEP, lymphatic endothelial cells, Chondrocytes, pDC, endothelial cells, Myocytes and CD4+ central memory T-cells infiltrated less (Figure 5B andSupplementary Table 8-9). The infiltration of dendritic cells (DC) was positively correlated with the expression of RIPK2 and the infiltration of Mast cells was negatively correlated with the expression of RIPK2 in dataset GSE32063 (Figure 5C and Supplementary Table 8-9). In dataset GSE63885, melanocyte's infiltration was high when RIPK2 had higher expression, while the infiltration of neurons and HSC was low (Figure 5D and Supplementary Table 8-9). 


\section{Discussion}

Serous ovarian cancer, one type of epithelial ovarian cancer, was conventionally treated by cytoreductive surgery and chemotherapy which was based on platinum and taxol(26). However, many patients died of chemoresistance gained during platinum and taxol treatment. While platinum resistance has gained more attention in ovarian cancer related studies, there is still a lack of detailed comprehension about potential biomarkers associated with taxol-resistance in ovarian cancer treatment. In this study, bioinformatic methods were used and a total of 103 DEGs (54 up-regulated 49 down-regulated) were found as the intersection of DEGs identified in 4 GEO datasets of taxol-sensitive and taxol-resistant cell lines. ISG15, SNCA, RIPK2, PLCG2, RHOU, TRIB2 and ELP2 influenced OS and PFI of ovarian cancer patients in TCGAOV dataset, while RIPK2 also affects the OS and PFI of ovarian cancer patients treated with taxol in datasets GSE30161, GSE32062 and GSE63885. Thus, it was validated by combining DEG analysis and survival analysis that higher expression of RIPK2 may lead to taxol resistance in serous ovarian cancer. The reliability of RIPK2 as a marker of taxol resistance was further verified when we divided TCGA-OV cohort to four groups, which were based on if patients were treated with or without taxol while had high or low RIPK2 expression. The OS of patients who had high expression of RIPK2 and were treated by taxol were significantly shorter than those with lower expression of RIPK2, which might suggest that patients with high expression of RIPK2 were tend to be sensitive to taxol treatment. This was confirmed when we found that the expression of RIPK2 was positively related to the IC50 to taxol of multiple ovarian cancer cell lines using data from CCLE database.

RIPK2 belongs to the family of Receptor-interacting protein (RIP) kinases and serves as a key molecule regulating inflammatory signaling and cell-death pathways(27). RIPK2 mediated signalling responses is initiated by the bacterial-sensing pattern recognition receptors nucleotide-binding oligomerization domain-containing proteins 1 and 2 (NOD1/2). Previous studies have shown that RIPK2 might be responsible for the chronic inflammation of Inflammatory Bowel Disease (IBD) $(28,29)$, and the high level of RIPK2 expression was associated with advanced tumor and metastasis of inflammatory breast cancer(30). It has also been reported that RIPK2 polymorphisms were related to tumor infiltration stages, lymph node metastasis and survival in urothelial bladder cancer(31) and the susceptibility of gastric cancer (32).

To probe the roles of RIPK2 in taxol resistance of serous ovarian cancer, we have identified its coexpressed genes in samples of patients treated by taxol from TCGA-OV dataset. It was found that the biological process involved in co-expressed genes were mainly the regulation of protein localization to membrane and the positive regulation of cellular protein localization, type I interferon production, nuclear factor kappa-light-chain-enhancer of activated B cells (NF-KB) transcription factor activity and cytokine production. The PPI network analysis showed that RIPK2 positively co-expressed with nuclear factor kappa B subunit 1 (NFKB1), baculoviral IAP repeat containing 2 (BIRC2) etc. The pathway analysis of RIPK2 co-expressed genes also demonstrated that these genes took part in positive regulation of NF-KB transcription factor activity. This might suggest that RIPK2 participated in taxol-resistant ovarian cancer by activating NF-KB mediated transcription (33). 
Recently, cancer-associated gene alterations had been studied in pan-cancer databases, revealing that CNA might be a marker in cancer genome for somatic genomic mutation that leads to tumorigenesis. Oncogenic driver genes with increased copy number and expression can be used as potential drug targets for tumor targeted therapy(34). In our study, it was found that RIPK2 alteration occurred in $10 \%$ ovarian cancer patients who were treated with taxol. Furthermore, mRNA upregulation occurred most frequently and the major type of genomic alteration was amplification, which resulted in high expression of RIPK2 compared with the diploid group, gain or shallow depletion group. We also found that the copy number frequency of OSGIN2, NBN differed in RIPK2-altered and -unaltered patients while these genes also coexpressed with RIPK2 in ovarian cancer. Rohit Mehra et al.(35) found that RIPK2-OSGIN2 gene fusion could happen in patients suffering of Primary clear-cell adenocarcinoma of the urethra. However, there was no reports on RIPK2 related gene alterations in ovarian cancer. The mutation frequency of 10 genes, including ASAP1, AGTPBP1, FZD7 etc., differed when RIPK2 differed in expression. ASAP1 and RIPK2 were reported as hub proteins of inflammatory bowel disease and colorectal cancer; and ASAP1 expression might be associated with pulmonary and bladder neoplasm diseases(36). Therefore, our research suggested that $A S A P 1$ mutation might be related to RIPK2 alteration and thus be associated with taxol resistance of ovarian cancer.

Immune infiltration is reported to have a tight association with tumor progress and prognosis, which could be as markers for drug responses in multiple tumors(37). Ellen L Goode et al. (38) has reported that CD8+ T lymphocytes infiltration were significantly associated with longer overall survival in HGSOCs. In this study, ssGSEA method was applied by using R package "xCell" and the correlation of infiltration of immune cells to RIPK2 was analyzed. The infiltration of Neuron was found to negatively correlated with RIPK2 expression in three datasets and MEP, mast cells were found in two datasets. The expression of DC, CD4+ memory T cells and CLP was positively correlated with RIPK2 in two datasets. These results might suggest that high expression of RIPK2 could influence the tumor microenvironment by affecting the infiltration of neuron, DC, CD4+ memory T cells and CLP.

\section{Conclusion}

In summary, we found that high expression of RIPK2 might be associated with the chemoresistance of taxol in serous ovarian cancer by identifying the intersected DEGs and applying survival analysis in multiple datasets. Our results suggested that RIPK2 up-regulation was likely to cause resistance to taxol through controlling the infiltration of immune cells. The expression of RIPK2 was significantly co-related to the expression of $N F K B 1$, indicating that taxol resistance might be related to the activation of NOD1/RIPK2/NF-KB inflammatory pathways. However, further experimental validation is required for confirmation of these results. These findings provided novel insights about using RIPK2 as a biomarker for taxol resistance and its possible mechanisms, which paved the way towards possible solution to taxol resistance in serous ovarian cancer.

\section{Abbreviations}


ABCB1 ATP binding cassette subfamily B member 1

AGTPBP1 ATP/GTP binding protein 1

ASAP1 ADP-ribosylation factor (Arf) GTPase-activating protein 1

BP biological process

CALB1 calbindin 1

cBioProtal cBio Cancer Genomics Portal

CC cellular component

CCLE Cancer Cell Line Encyclopedia

CNA copy number alteration

CNV copy number variation

DEGs differentially expressed genes

ELP3 elongator acetyltransferase complex subunit 3

EPHA7 ephrin receptor A7

FZD7 frizzled class receptor 7

GEO Gene Expression Omnibus

GO gene ontology

HERC5 HECT and RLD domain containing E3 ubiquitin protein ligase 5

HGSOC high-grade serous ovarian cancer

HMGN5 high mobility group nucleosome binding domain 5

ISG15 interferon stimulated gene 15

KEGG Kyoto Encyclopedia of Genes and Genomes

KIAA0232 PATJ crumbs cell polarity complex component

LGSOC low-grade serous ovarian cancer

LIPG Endothelial lipase

MAP3K10 Mitogen-Activated Protein Kinase Kinase Kinase 10

MCTP1 multiple C2 and transmembrane domain containing 1

MELK maternal embryonic leucine zipper kinase

MF molecular function

NBN nibrin 


\begin{tabular}{|c|c|}
\hline OS & overall survival \\
\hline OSGIN2 & oxidative stress induced growth inhibitor family member 2 \\
\hline PATJ & Pals-associated tight junction protein \\
\hline PCDH9 & protocadherin 9 \\
\hline PDAP1 & platelet-derived growth factor A-associated protein 1 \\
\hline PFI & progress-free interval \\
\hline PLCG2 & Phospholipase C gamma 2 \\
\hline PLK2 & polo-like kinase 2 \\
\hline PPI & Protein-protein interaction network \\
\hline PTPRK & protein tyrosine phosphatase receptor type $\mathrm{K}$ \\
\hline RAB2A & Ras-Related Protein Rab-2A \\
\hline RHOU & ras homolog family member $U$ \\
\hline RIPK2 & receptor interacting serine/threonine kinase 2 \\
\hline RUNDC3B & RUN domain containing 3B \\
\hline SEMA3A & semaphorin $3 \mathrm{~A}$ \\
\hline SFN & Stratifin \\
\hline SIK2 & salt inducible kinase \\
\hline SNCA & synuclein alpha \\
\hline SOC & Serous ovarian cancer \\
\hline ssGSEA & single-sample Gene Set Enrichment Analysis \\
\hline SYK & spleen tyrosine kinase \\
\hline TCGA & the Cancer Genome Atlas \\
\hline TRIB2 & tribbles pseudokinase 2 \\
\hline TUBB3 & tubulin beta 3 class III \\
\hline XIRP1 & xin actin binding repeat containing 1 \\
\hline
\end{tabular}

\section{Declarations}

Ethics approval and consent to participate No ethics approval was required for this work. All utilized public data sets were generated by others who obtained ethical approval. 
Availability of data and materials The datasets generated and/or analyzed in this study are available in the TCGA repository

(https://portal.gdc.cancer.gov/repository?facetTab=cases)

and GEO repository (https://www.ncbi.nlm.nih.gov/geo/query/acc.cgi?acc=GSE58840, https://www.ncbi.nlm.nih.gov/geo/query/acc.cgi?acc=GSE60335, https://www.ncbi.nlm.nih.gov/geo/query/acc.cgi?acc=GSE58878, https://www.ncbi.nlm.nih.gov/geo/query/acc.cgi?acc=GSE26465,

https://www.ncbi.nlm.nih.gov/geo/query/acc.cgi?acc=GSE73935, https://www.ncbi.nlm.nih.gov/geo/query/acc.cgi?acc=GSE54772, https://www.ncbi.nlm.nih.gov/geo/query/acc.cgi?acc=GSE30161, https://www.ncbi.nlm.nih.gov/geo/query/acc.cgi?acc=GSE32063, https://www.ncbi.nlm.nih.gov/geo/query/acc.cgi?acc=GSE63885).

Competing interests The authors declare that they have no competing interests. None of the authors have financial or non-financial competing interests with this work.

Authors' contributions All authors contributed to the study conception and design. Yuqing Shen and Hui Lin performed data collection and computational analysis and wrote the first draft of the manuscript. Kelie Chen, Wanzhong Ge, Dajing Xia, Yihua Wu and Weiguo Lu edited and reviewed the manuscript. All authors read and commented on the manuscript and agreed upon a final version together.

Funding This research was supported by grants from the National Natural Science Foundation of China (Grants 31005808A11 12).

Acknowledgements Not applicable.

\section{References}

1. van Zyl B, Tang D, Bowden NA: Biomarkers of platinum resistance in ovarian cancer: what can we use to improve treatment. Endocrine-related cancer 2018, 25(5):R303-r318.

2. McPherson A, Roth A, Laks E, Masud T, Bashashati A, Zhang AW, Ha G, Biele J, Yap D, Wan A et al: Divergent modes of clonal spread and intraperitoneal mixing in high-grade serous ovarian cancer. Nature genetics 2016, 48(7):758-767.

3. Torre LA, Trabert B, DeSantis CE, Miller KD, Samimi G, Runowicz CD, Gaudet MM, Jemal A, Siegel RL: Ovarian cancer statistics, 2018. CA: a cancer journal for clinicians 2018, 68(4):284-296.

4. Colombo PE, Fabbro M, Theillet C, Bibeau F, Rouanet P, Ray-Coquard I: Sensitivity and resistance to treatment in the primary management of epithelial ovarian cancer. Critical reviews in oncology/hematology 2014, 89(2):207-216. 
5. Morgan RJ, Jr., Alvarez RD, Armstrong DK, Burger RA, Castells M, Chen LM, Copeland L, Crispens MA, Gershenson D, Gray H et al: Ovarian cancer, version 3.2012. Journal of the National Comprehensive Cancer Network : JNCCN2012, 10(11):1339-1349.

6. Kampan NC, Madondo MT, McNally OM, Quinn M, Plebanski M: Paclitaxel and Its Evolving Role in the Management of Ovarian Cancer. BioMed research international 2015, 2015:413076.

7. Roque DM, Bellone S, Buza N, Romani C, Cocco E, Bignotti E, Ravaggi A, Rutherford TJ, Schwartz PE, Pecorelli $S$ et al: Class III $\beta$-tubulin overexpression in ovarian clear cell and serous carcinoma as a maker for poor overall survival after platinum/taxane chemotherapy and sensitivity to patupilone. American journal of obstetrics and gynecology 2013, 209(1):62.e61-69.

8. English DP, Menderes G, Black J, Schwab CL, Santin AD: Molecular diagnosis and molecular profiling to detect treatment-resistant ovarian cancer. Expert review of molecular diagnostics 2016, 16(7):769782.

9. Yu Y, Gaillard S, Phillip JM, Huang TC, Pinto SM, Tessarollo NG, Zhang Z, Pandey A, Wirtz D, Ayhan A et al: Inhibition of Spleen Tyrosine Kinase Potentiates Paclitaxel-Induced Cytotoxicity in Ovarian Cancer Cells by Stabilizing Microtubules. Cancer cell 2015, 28(1):82-96.

10. Januchowski R, Sterzyńska K, Zawierucha P, Ruciński M, Świerczewska M, Partyka M, BednarekRajewska K, Brązert M, Nowicki M, Zabel M et al: Microarray-based detection and expression analysis of new genes associated with drug resistance in ovarian cancer cell lines. Oncotarget 2017, 8(30):49944-49958.

11. Hu Y, Zeng Q, Li C, Xie Y: Expression profile and prognostic value of SFN in human ovarian cancer. Bioscience reports 2019, 39(5).

12. Kohler RS, Kettelhack H, Knipprath-Mészaros AM, Fedier A, Schoetzau A, Jacob F, HeinzelmannSchwarz V: MELK expression in ovarian cancer correlates with poor outcome and its inhibition by OTSSP167 abrogates proliferation and viability of ovarian cancer cells. Gynecologic oncology 2017, 145(1):159-166.

13. Davis S, Meltzer PS: GEOquery: a bridge between the Gene Expression Omnibus (GEO) and BioConductor. Bioinformatics (Oxford, England) 2007, 23(14):1846-1847.

14. Ritchie ME, Phipson B, Wu D, Hu Y, Law CW, Shi W, Smyth GK: limma powers differential expression analyses for RNA-sequencing and microarray studies. Nucleic acids research 2015, 43(7):e47.

15. Chen H, Boutros PC: VennDiagram: a package for the generation of highly-customizable Venn and Euler diagrams in R. BMC bioinformatics 2011, 12:35.

16. Terry M. Therneau PMG: Modeling Survival Data: Extending the Cox Model. Springer, New York ISBN 0-387-98784-32000.

17. Alboukadel Kassambara MK, Przemyslaw Biecek: survminer: Drawing Survival Curves using 'ggplot2'. R package version 0.4.6.https://CRANR-projectorg/package=survminer 2019.

18. Barretina J, Caponigro G, Stransky N, Venkatesan K, Margolin AA, Kim S, Wilson CJ, Lehár J, Kryukov GV, Sonkin D et al: The Cancer Cell Line Encyclopedia enables predictive modelling of anticancer drug sensitivity. Nature 2012, 483(7391):603-607. 
19. Taiyun Wei VS: R package "corrplot": Visualization of a Correlation Matrix (Version 0.84). 2017.

20. Yu G, Wang LG, Han Y, He QY: clusterProfiler: an R package for comparing biological themes among gene clusters. Omics : a journal of integrative biology 2012, 16(5):284-287.

21. Wickham. H: ggplot2: Elegant Graphics for Data Analysis. Springer-Verlag New York 2016.

22. Szklarczyk D, Gable AL, Lyon D, Junge A, Wyder S, Huerta-Cepas J, Simonovic M, Doncheva NT, Morris JH, Bork $\mathrm{P}$ et al: STRING v11: protein-protein association networks with increased coverage, supporting functional discovery in genome-wide experimental datasets. Nucleic acids research 2019, 47(D1):D607-d613.

23. Lopes CT, Franz M, Kazi F, Donaldson SL, Morris Q, Bader GD: Cytoscape Web: an interactive webbased network browser. Bioinformatics (Oxford, England) 2010, 26(18):2347-2348.

24. Aran D, Hu Z, Butte AJ: xCell: digitally portraying the tissue cellular heterogeneity landscape. Genome biology 2017, 18(1):220.

25. Gao J, Aksoy BA, Dogrusoz U, Dresdner G, Gross B, Sumer SO, Sun Y, Jacobsen A, Sinha R, Larsson E et al: Integrative analysis of complex cancer genomics and clinical profiles using the cBioPortal. Science signaling 2013, 6(269):pl1.

26. Freimund AE, Beach JA, Christie EL, Bowtell DDL: Mechanisms of Drug Resistance in High-Grade Serous Ovarian Cancer. Hematology/oncology clinics of North America 2018, 32(6):983-996.

27. He S, Wang X: RIP kinases as modulators of inflammation and immunity. Nature immunology 2018, 19(9):912-922.

28. Garcia-Carbonell R, Yao SJ, Das S, Guma M: Dysregulation of Intestinal Epithelial Cell RIPK Pathways Promotes Chronic Inflammation in the IBD Gut. Frontiers in immunology 2019, 10:1094.

29. Topal Y, Gyrd-Hansen M: RIPK2 NODs to XIAP and IBD. Seminars in cell \& developmental biology 2020.

30. Zare A, Petrova A, Agoumi M, Amstrong H, Bigras G, Tonkin K, Wine E, Baksh S: RIPK2: New Elements in Modulating Inflammatory Breast Cancer Pathogenesis. Cancers 2018, 10(6).

31. Guirado M, Gil H, Saenz-Lopez P, Reinboth J, Garrido F, Cozar JM, Ruiz-Cabello F, Carretero R: Association between C130RF31, NOD2, RIPK2 and TLR10 polymorphisms and urothelial bladder cancer. Human immunology 2012, 73(6):668-672.

32. Ota M, Tahara T, Otsuka T, Jing W, Nomura T, Hayashi R, Shimasaki T, Nakamura M, Shibata T, Arisawa T: Association between receptor interacting serine/threonine kinase 2 polymorphisms and gastric cancer susceptibility. Oncology letters 2018, 15(3):3772-3778.

33. Mares A, Miah AH, Smith IED, Rackham M, Thawani AR, Cryan J, Haile PA, Votta BJ, Beal AM, Capriotti $C$ et al: Extended pharmacodynamic responses observed upon PROTAC-mediated degradation of RIPK2. Communications biology 2020, 3(1):140.

34. Calabrese C, Davidson NR, Demircioğlu D, Fonseca NA, He Y, Kahles A, Lehmann KV, Liu F, Shiraishi Y, Soulette CM et al: Genomic basis for RNA alterations in cancer. Nature 2020, 578(7793):129-136. 
35. Mehra R, Vats P, Kalyana-Sundaram S, Udager AM, Roh M, Alva A, Pan J, Lonigro RJ, Siddiqui J, Weizer $\mathrm{A}$ et al: Primary urethral clear-cell adenocarcinoma: comprehensive analysis by surgical pathology, cytopathology, and next-generation sequencing. The American journal of pathology 2014, 184(3):584-591.

36. Al-Mustanjid M, Mahmud SMH, Royel MRI, Rahman MH, Islam T, Rahman MR, Moni MA: Detection of molecular signatures and pathways shared in inflammatory bowel disease and colorectal cancer: A bioinformatics and systems biology approach. Genomics 2020, 112(5):3416-3426.

37. Li B, Severson E, Pignon JC, Zhao H, Li T, Novak J, Jiang P, Shen H, Aster JC, Rodig S et al: Comprehensive analyses of tumor immunity: implications for cancer immunotherapy. Genome biology 2016, 17(1):174.

38. Goode EL, Block MS, Kalli KR, Vierkant RA, Chen W, Fogarty ZC, Gentry-Maharaj A, Tołoczko A, Hein A, Bouligny AL et al: Dose-Response Association of CD8+ Tumor-Infiltrating Lymphocytes and Survival Time in High-Grade Serous Ovarian Cancer. JAMA oncology 2017, 3(12):e173290.

\section{Figures}
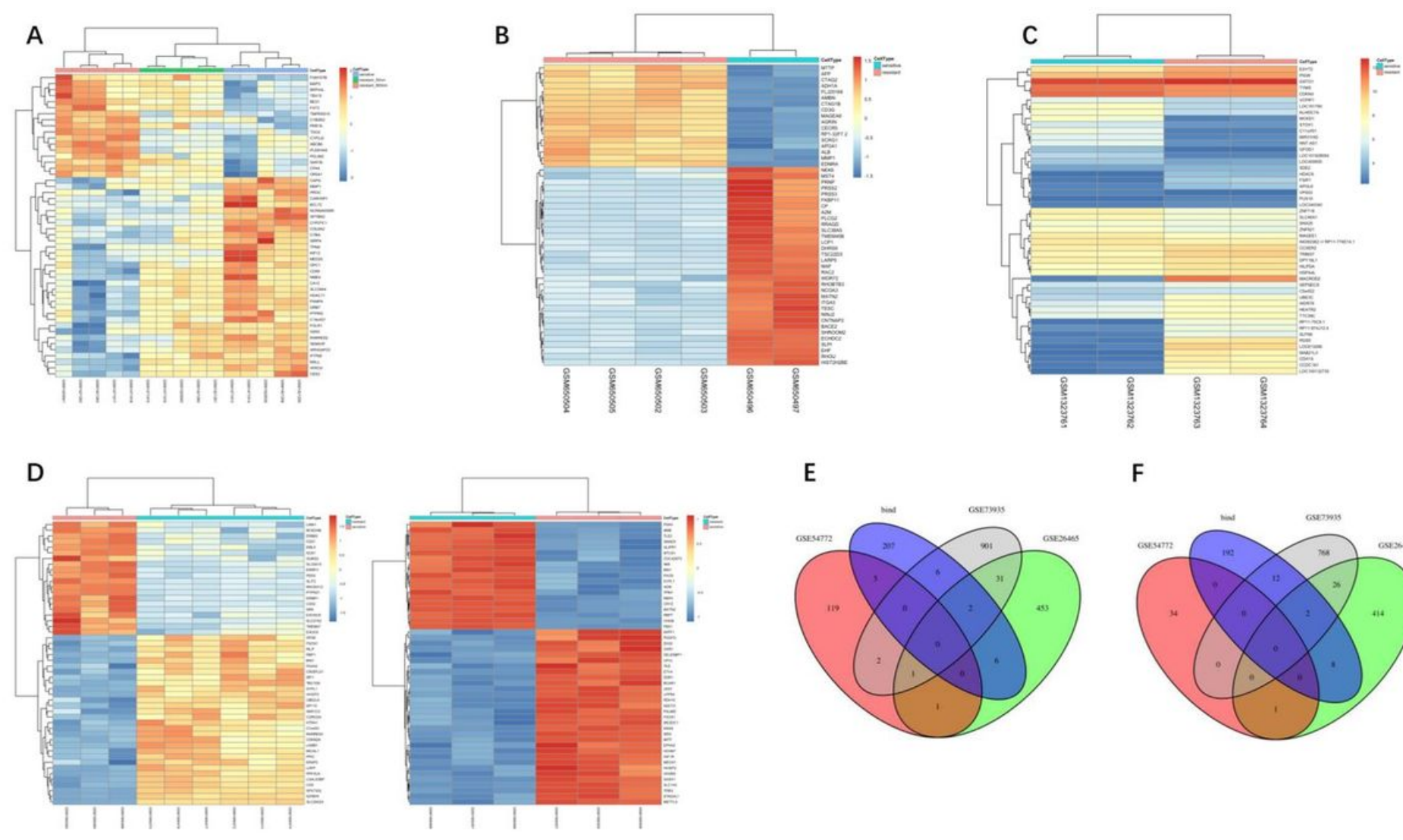

F

\section{Figure 1}

Venn diagram and heatmaps for differentially expressed genes (DEGs) in mRNA sequencing datasets. (A) heatmaps for DEGs in dataset GSE58840, GSE60335 and GSE58878. (B) heatmaps for DEGs in dataset GSE26465. (C) heatmaps for DEGs in dataset GSE73935. (D) heatmaps for DEGs in dataset GSE54772. 
(E)Venn diagram showing the intersection of the up-regulated DEGs from datasets GSE58840, GSE60335, GSE58878, GSE26465, GSE73935 and GSE54772. (F)Venn diagram showing the intersection of the down-regulated DEGs from datasets GSE58840, GSE60335, GSE58878, GSE26465, GSE73935 and GSE54772.

A
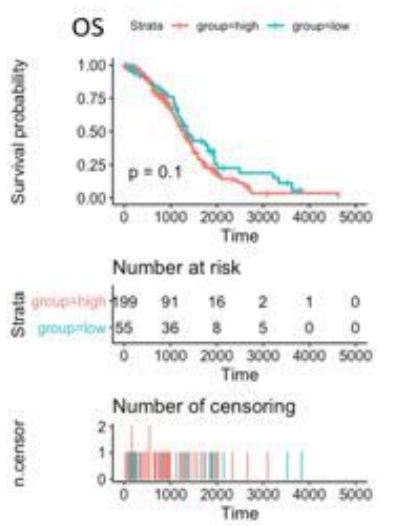

$\mathrm{C}$

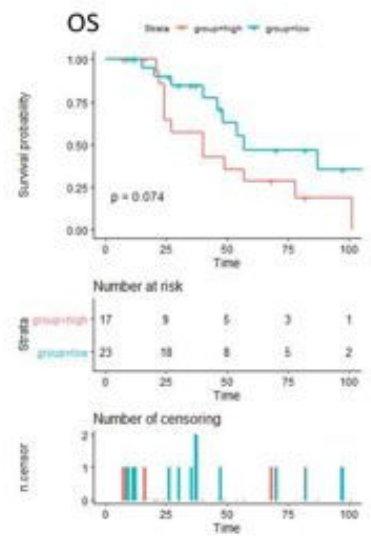

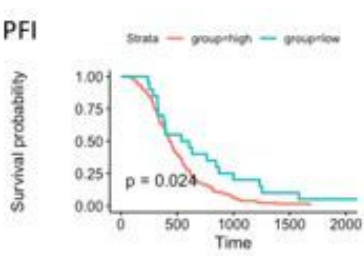

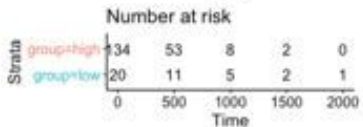

Number of censoring
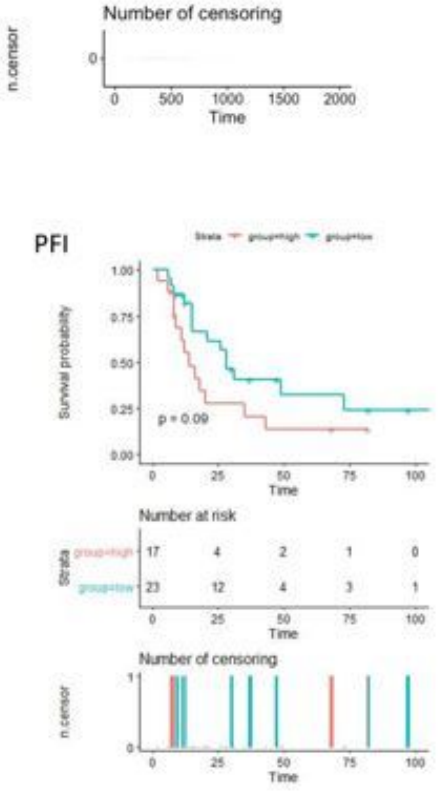

$E$

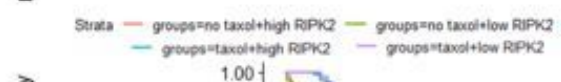

है
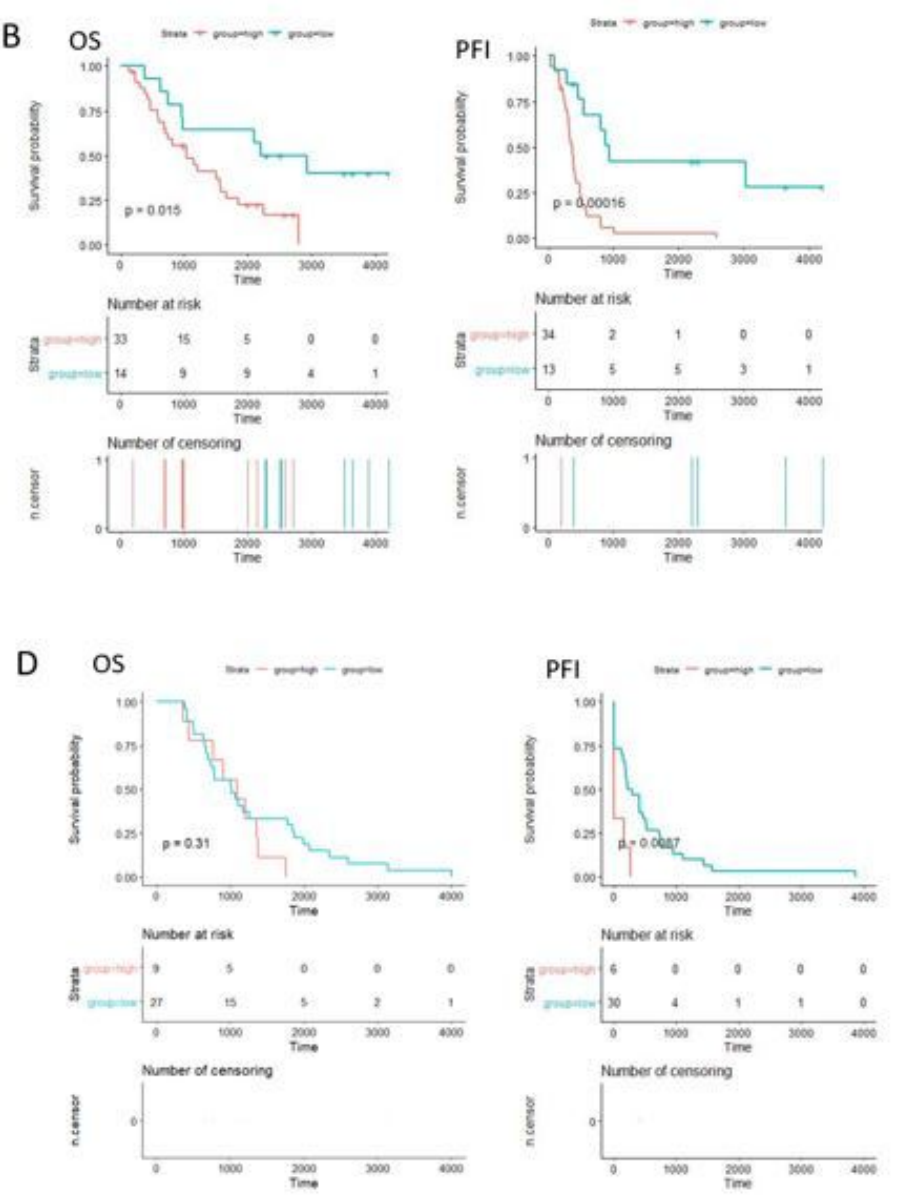
progress free interval (PFI) in RIPK2 high and low expression group in GSE30161 dataset. (C) Overall survival (OS) and progress free interval (PFI) in RIPK2 high and low expression group in GSE32063 dataset. (D) Overall survival (OS) and progress free interval (PFI) in RIPK2 high and low expression group in GSE63885 dataset. (E) Overall survival (OS) of groups defined by RIPK2 expression and taxol usage in TCGA-OV cohort. The numbers below the figures denote the number of patients at risk in each group.
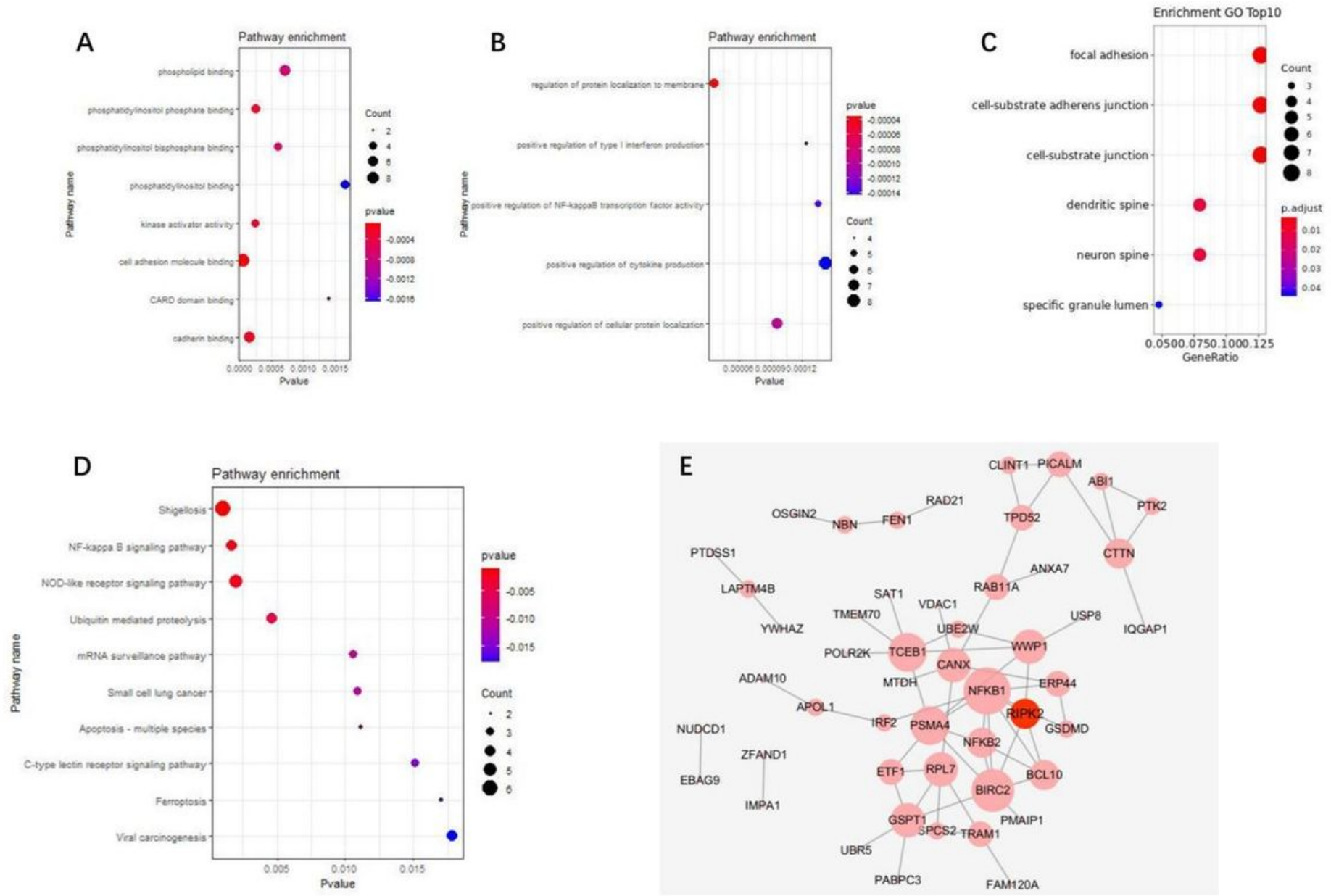

\section{Figure 3}

Gene Ontology (GO), Kyoto Encyclopedia of Genes and Genomes(KEGG) enrichment analyses and protein-protein network(PPI) analysis of co-expressed genes of RIPK2 in serous ovarian cancer patients treated with taxol. (A) molecular function of GO terms. (B) Biological process of $\mathrm{GO}$ terms. (C) cellular component of GO terms. (D) KEGG enrichment of genes co-expressed with RIPK2. The horizontal axis represents the number of DEGs under the GO/KEGG term and the sizes of the dots represents the number of genes located in the functional area. (E) PPI network generated by "STRING" database and visualized by Cytoscape. Nodes represent co-expressed genes and edges represent PPIs. 

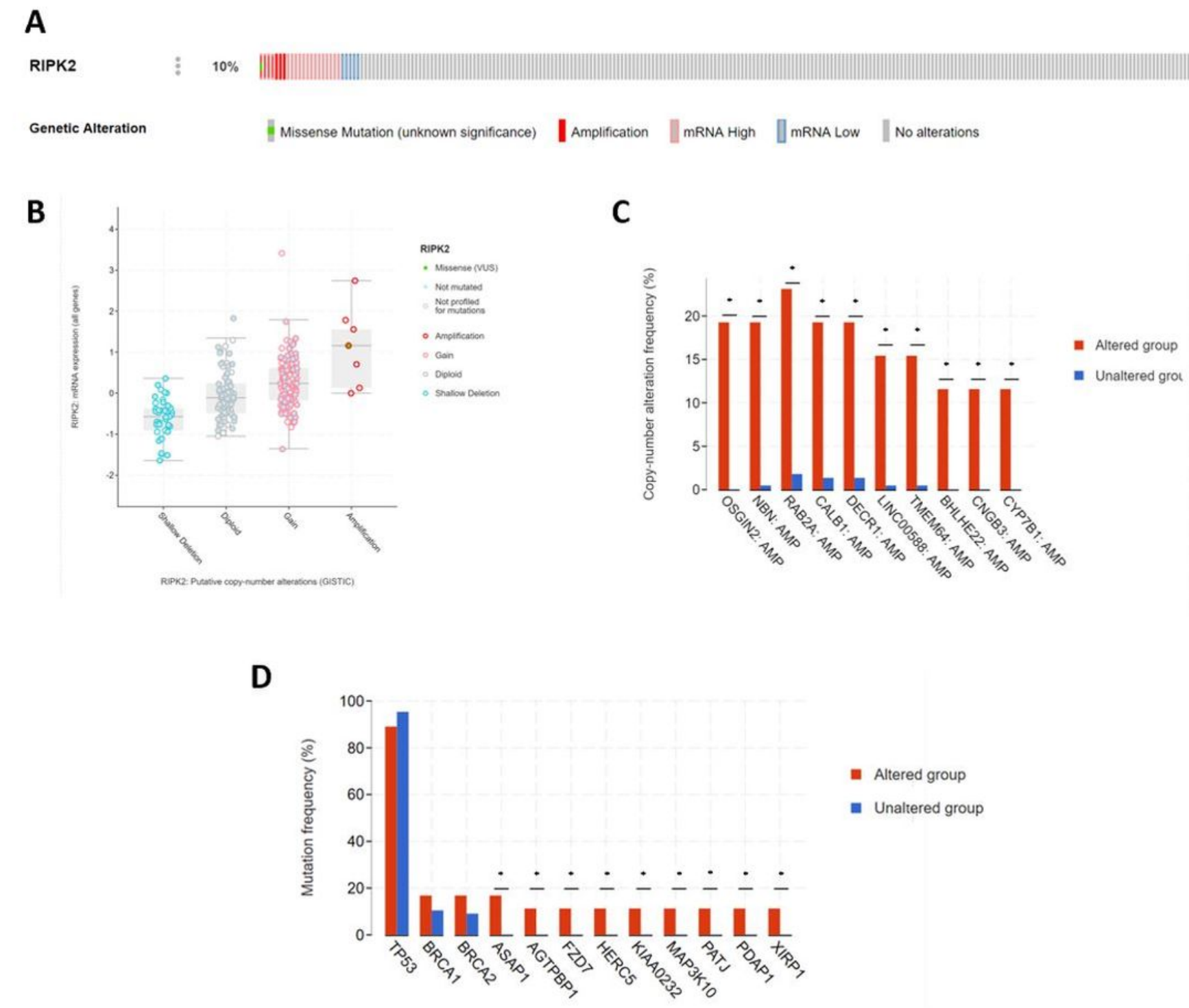

\section{Figure 4}

RIPK2 genomic alterations in ovarian cancer (cBioPortal). (A) OncoPrint of RIPK2 alterations in TCGA-OV cohort. Different types of genetic alterations are highlighted in different colors. B) the relationship of copy number alterations and mRNA expression of RIPK2. C) difference of genetic mutations in RIPK2 altered and unaltered group. Tumor protein p53 (TP53), BReast CAncer gene 1 (BRCA1), BReast CAncer gene 2 (BRCA2) and 10 other genes with the most significant $p$ values were shown. D) copy-number change. 10 genes with the most significant $p$ values were shown. ${ }^{\star} p<0.01$. 

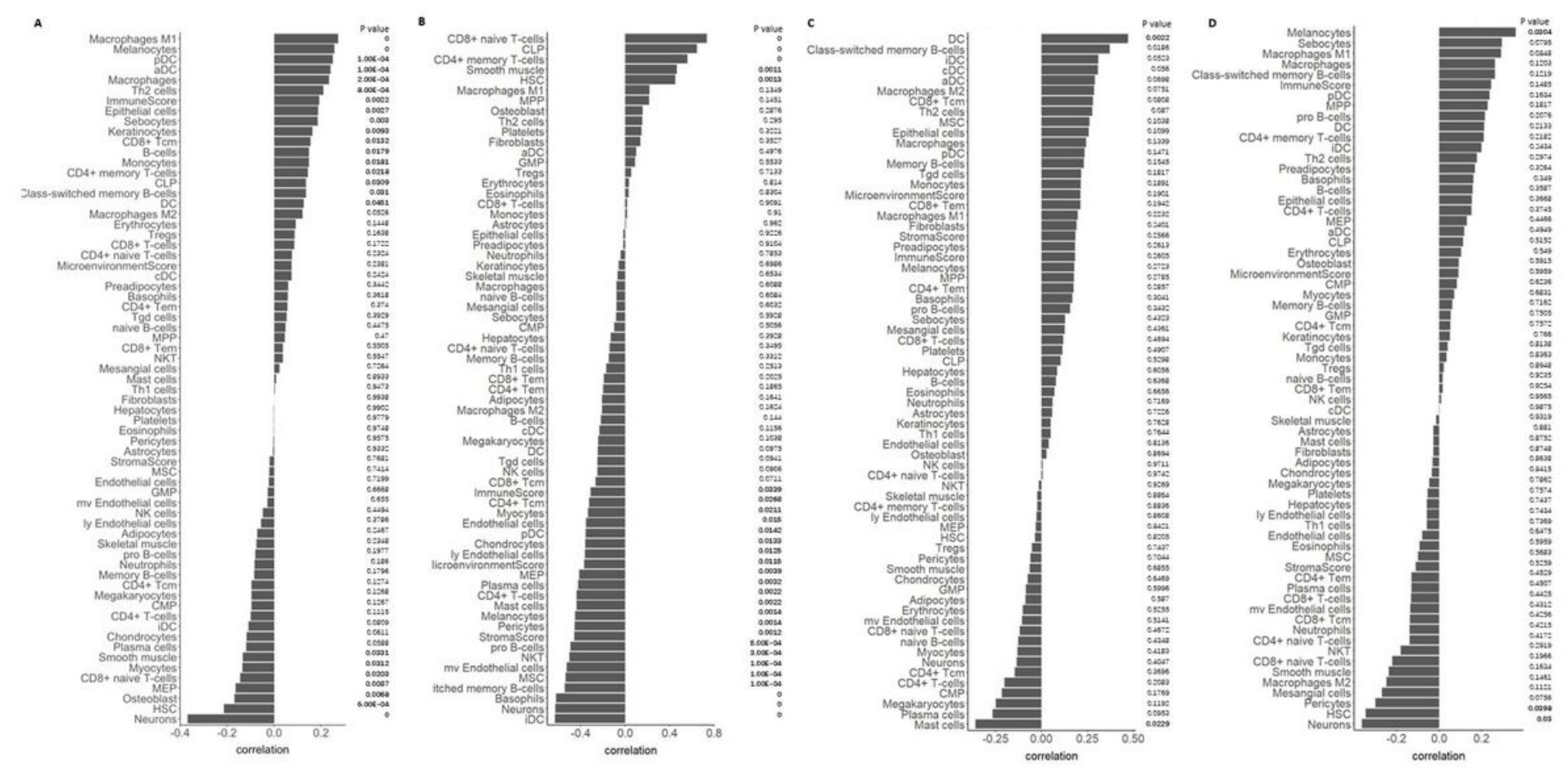

\section{Figure 5}

Correlation between RIPK2 expression and immune infiltration (A) Correlation between RIPK2 expression and infiltrating immune cells in TCGA-OV dataset. (B) Correlation between RIPK2 expression and infiltrating immune cells in GSE30161 dataset. (C) Correlation between RIPK2 expression and infiltrating immune cells in GSE32063 dataset. (D) Correlation between RIPK2 expression and infiltrating immune cells in GSE63885 dataset. $\mathrm{p}<0.05$ was considered statistically significant. MPP, Multipotent rogenitors; CD8+ Tem, CD8+ effector memory T-cells; CMP, Common myeloid progenitors; GMP, Granulocytemacrophage progenitors; MEP, Megakaryocyte-erythroid progenitors; Tregs, Regulatory T-cells; HSC, Hematopoietic stem cells; CD4+ Tcm, CD4+ central memory T-cells; mv Endothelial cells, Microvascular endothelial cells; CD4+ Tem, CD4+ effector memory T-cells; CD8+ Tcm, CD8+ central memory T-cells; ly Endothelial cells, Lymphatic endothelial cells; MSC, Mesenchymal stem cells; aDC, Activated dendritic cells; $\mathrm{CDC}$, Xonventional dendritic cells; pDC, Plasmacytoid dendritic cells; iDC, Immature dendritic cells; Th2 cells, Type 2 T-helper cells; CLP, Common lymphoid progenitors; Th1 cells, Type 1 T-helper cells; NKT, Natural killer T-cells; Tgd cells, Gamma delta T-cells.

\section{Supplementary Files}

This is a list of supplementary files associated with this preprint. Click to download.

- SupplementaryTable1DEGsof4datasets.xIsx

- SupplementaryTable2IDsofsampleswithtaxoltreatment.xlsx

- SupplementaryTable3relationshipofIC50withRIPK2.csv 
- SupplementaryTable4genescoexpressedwithRIPK2.xIsx

- SupplementaryTable5GOKEGGandPPlanalysis.xIsx

- SupplementaryTable6relationshipofCNAwithRIPK2.csv

- SupplementaryTable7relationshipofmutationwithRIPK2.csv

- SupplementaryTable8immuneinfiltration.xIsx

- SupplementaryTable9correlationRIPK2immuneinfiltration.csv

- supp.figure1to6.docx 